Fabiano Petronetto do Carmo

\title{
A Equação de Poisson e a Decomposição de Helmholtz-Hodge com Operadores SPH
}

Tese de Doutorado

Tese apresentada ao Programa de Pós-graduação em Matemática Aplicada do Departamento de Matemática da PUCRio como requisito parcial para obtenção do título de Doutor em Matemática Aplicada

Orientador : Prof. Geovan Tavares dos Santos Co-Orientador: Prof. Welles Antonio Martinez Morgado 
Fabiano Petronetto do Carmo

\section{A Equação de Poisson e a Decomposição de Helmholtz-Hodge com Operadores SPH}

Tese apresentada ao Programa de Pós-graduação em Matemática Aplicada do Departamento de Matemática do Centro Técnico Científico da PUC-Rio como requisito parcial para obtenção do título de Doutor em Matemática Aplicada. Aprovada pela Comissão Examinadora abaixo assinada.

Prof. Geovan Tavares dos Santos Orientador

Departamento de Matemática - PUC-Rio

Prof. Welles Antonio Martinez Morgado Co-Orientador Departamento de Física - PUC-Rio

Prof. Antonio Castelo Filho Instituto de Ciências Matemáticas e de Computação - USP

Prof. Carlos Eduardo Aguiar Instituto de Física - UFRJ

Prof. Luiz Carlos Pacheco Rodrigues Velho Laboratório Visgraf — IMPA

Prof. Marcio da Silveira Carvalho Departamento de Engenharia Mecânica - PUC-Rio

Prof. Hélio Côrtes Vieira Lopes Departamento de Matemática — PUC-Rio

Prof. Thomas Lewiner Departamento de Matemática — PUC-Rio

Prof. José Eugênio Leal Coordenador Setorial do Centro Técnico Científico - PUC-Rio 
Todos os direitos reservados. É proibida a reprodução total ou parcial do trabalho sem autorização da universidade, do autor e do orientador.

\section{Fabiano Petronetto do Carmo}

Bacharel em Matemática na Universidade Federal do Espírito Santo (Vitória, Brasil) em 2002. Mestre em Matemática, opção em Computação Gráfica, no Instituto Nacional de Matemática Pura e Aplicada (Rio de Janeiro, Brasil) em 2004.

Ficha Catalográfica

do Carmo, Fabiano Petronetto

A Equação de Poisson e a Decomposição de HelmholtzHodge com Operadores SPH / Fabiano Petronetto do Carmo; orientador: Geovan Tavares dos Santos; co-orientador: Welles Antonio Martinez Morgado. - Rio de Janeiro : PUC-Rio, Departamento de Matemática, 2008.

v., 132 f: il. ; $29,7 \mathrm{~cm}$

1. Tese (doutorado) - Pontifícia Universidade Católica do Rio de Janeiro, Departamento de Matemática.

Inclui referências bibliográficas.

1. Matemática - Tese. 2. Dinâmica dos Fluidos Computacionais. 3. Método de Partículas. 4. Smoothed Particle Hydrodynamics. 5. Equação de Poisson. 6. Escoamentos Bifásicos. 7. Decomposição de Helmholtz-Hodge. I. dos Santos, Geovan Tavares. II. Morgado, Welles Antonio Martinez. III. Pontifícia Universidade Católica do Rio de Janeiro. Departamento de Matemática. IV. Título. 


\section{Agradecimentos}

À minha família, agradeço todo o amor, carinho, compreensão e respeito.

À minha querida noiva Cíntia (minha pequena), que sempre me estimula a crescer científica, ética, profissional e pessoalmente. A quem esteve comigo durante toda a realização desse sonho: o meu muito obrigado por existir.

Ao Professor Geovan Tavares, mais que um orientador, um amigo que sempre compartilhou entusiasticamente de todo o meu aprendizado. Sempre ao meu lado, incentivando e ajudando a superar todas as dificuldades.

Aos Professores Thomas Lewiner e Hélio Lopes, também grandes amigos, pela atenção e inúmeras sugestões que enriqueceram o meu conhecimento.

À família do ap906: Afonso, Rener, Thiago e Etereldes. Em especial ao "irmão" Thiago, companheiro de quarto por 6 anos, e ao amigo Afonso que compartilhamos a aventura do doutorado por 4 anos.

Aos colegas que encontrei durante esses 6 anos no Rio de Janeiro: Galeguinho, Galão, LG, Tanaka, Paulinho mão-de-quiabo, Brands, Fernando, Aninha, Francisco, Laier, Marquinhos e Lhaylha.

Aos funcionários do Departamento de Matemática da PUC-Rio pela atenção e disponibilidade para resolver todos os problemas de última hora.

À Faperj, Capes, Petrobrás e PUC-Rio pelos auxílios concedidos, o nosso pão de cada dia. 


\section{Resumo}

do Carmo, Fabiano Petronetto; dos Santos, Geovan Tavares; Morgado, Welles Antonio Martinez. A Equação de Poisson e a Decomposição de Helmholtz-Hodge com Operadores SPH. Rio de Janeiro, 2008. 132p. Tese de Doutorado - Departamento de Matemática, Pontifícia Universidade Católica do Rio de Janeiro.

A equação diferencial parcial de Poisson é de fundamental importância em várias áreas de pesquisa, dentre elas: matemática, física e engenharia. Para resolvê-la numericamente utilizam-se vários métodos, tais como os já tradicionais métodos das diferenças finitas e dos elementos finitos. Este trabalho propõe um método para resolver a equação de Poisson, utilizando uma abordagem de sistema de partículas conhecido como SPH, do inglês Smoothed Particles Hydrodynamics. O método proposto para a solução da equação de Poisson e os operadores diferenciais discretos definidos no método SPH, chamados de operadores SPH, são utilizados neste trabalho em duas aplicações: na decomposição de campos vetoriais; e na simulação numérica de escoamentos de fluidos monofásicos e bifásicos utilizando a equação de Navier-Stokes.

\section{Palavras-chave}

Dinâmica dos Fluidos Computacionais. Método de Partículas. Smoothed Particle Hydrodynamics. Equação de Poisson. Escoamentos Bifásicos. Decomposição de Helmholtz-Hodge. 


\section{Abstract}

do Carmo, Fabiano Petronetto; dos Santos, Geovan Tavares; Morgado, Welles Antonio Martinez. Poisson Equation and the Helmholtz-Hodge Decomposition with SPH operators. Rio de Janeiro, 2008. 132p. PhD Thesis - Department of Mathematics, Pontifícia Universidade Católica do Rio de Janeiro.

Poisson's equation is of fundamental importance in many research areas in engineering and the mathematical and physical sciences. Its numerical solution uses several approaches among them finite differences and finite elements. In this work we propose a method to solve Poisson's equation using the particle method known as SPH (Smoothed Particle Hydrodynamics). The proposed method together with an accurate analysis of the discrete differential operators defined by SPH are applied in two related situations: the Hodge-Helmholtz vector field decomposition and the numerical simulation of the Navier-Stokes equations.

\section{Keywords}

Computational Fluid Dynamics. Particles Method. Smoothed Particle Hydrodynamics. Poisson Equation. Biphase Flows. Helmholtz-Hodge Decomposition. 


\section{Sumário}

1 Introdução $\quad 9$

2 Smoothed Particle Hydrodynamics $\quad \mathbf{1 5}$

$\begin{array}{lll}2.1 & \text { Conceitos básicos de SPH } & 15\end{array}$

$\begin{array}{lll}2.2 & \text { Representação Integral } & 16\end{array}$

2.3 Aproximação por Partículas 20

2.4 Núcleos: funções de base radial 22

$\begin{array}{lll}2.5 & \text { Partículas } & 26\end{array}$

3 Operadores SPH $\quad 29$

3.1 Operador Divergente SPH 29

3.2 Operador Gradiente SPH $\quad 40$

$\begin{array}{ll}3.3 & \text { Operador Laplaciano SPH }\end{array}$

4 Solução Numérica da Equação de Poisson Usando SPH 57

4.1 Equação de Poisson $\quad 57$

4.2 Equação de Poisson Discreta usando SPH 58

4.3 Matrizes Esparsas 61

4.4 Métodos iterativos 63

5 Decomposição de Helmholtz-Hodge $\quad 66$

5.1 Campos Vetoriais e Escalares $\quad 67$

5.2 Decomposição de Helmholtz-Hodge usando SPH 69

$\begin{array}{lll}5.3 & \text { Detecção automática de feições } & 77\end{array}$

$\begin{array}{lll}5.4 \text { Resultados } & 80\end{array}$

$6 \quad$ Escoamentos Incompressíveis $\quad 86$

6.1 Equações de Navier-Stokes $\quad 86$

6.2 Fluidos Incompressíveis 93

6.3 A Pressão em Escoamentos Incompressíveis 97

7 Simulação Numérica das Equações de Navier-Stokes usando SPH $\quad 99$

$\begin{array}{lll}7.1 & \text { SPH Navier-Stokes } & 101\end{array}$

$\begin{array}{lll}7.2 & \text { Resultados } & 108\end{array}$

8 Conclusão e Trabalhos Futuros $\quad 121$

$\begin{array}{ll}\text { Referências Bibliográficas } & 123\end{array}$ 


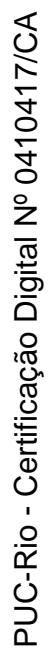

Para tudo existe um fim. Na vida, todo fim é um começo. 